\title{
Characterizing UVB-induced skin tumor process using optical coherence tomography
}

\author{
Shulian Wu, Zheng Huang, Yunxia Wang and Hui Li* \\ Key Lab of OptoElectronic Science and Technology for Medicine \\ Fujian Provincial Key Lab of Photonic Technology \\ College of Photonic and Electronic Engineering \\ Fujian Normal University \\ Fuzhou 350007, Fujian, P. R. China \\ *hli@fjnu.edu.cn
}

Received 30 August 2015

Accepted 29 October 2015

Published 23 December 2015

\begin{abstract}
The incidence of nonmelanoma skin cancer is rising worldwide. The major carcinogenic factor for most skin cancers is solar ultraviolet light, in particular, the chronic exposure to UVB $(280-320 \mathrm{~nm})$. In this study, the optical characteristics of skin canceration process induced by UVB were analyzed. Optical coherence tomography (OCT) was used for monitoring the morphologic changes and compared with histological analysis. Meanwhile, the optical property, such as the attenuation coefficient $\left(\mu_{\mathrm{t}}\right)$ was systematically extracted and analyzed. In addition, characteristic textures, including energy, entropy and correlation were revealed from OCT images. Results suggest that OCT is a useful tool for monitoring the process of UVB-induced skin cancer and changes of optical property during this process.
\end{abstract}

Keywords: Skin cancer; UVB; morphologic change; attenuation coefficient; texture.

\section{Introduction}

The skin cancer is the most common type of cancer and its incidence has been growing in an alarming rate worldwide mainly due to the destruction of the ozone layer in recent years. ${ }^{1-3}$ Skin cancer can lead to severe skin injury if incompletely removed with a wide margin of normal tissue, which resulted in adverse physical and psychological consequences to the patients. It is well known that excessive and long-term exposure to UVB is a main contributing factor of skin cancer. ${ }^{4,5}$

The gold standard for diagnosis of cutaneous diseases is still biopsy and histopathological examination. But this technique is invasive and timeconsuming. In recent years, many well-established noninvasive optical imaging methods (e.g., optical coherence tomography (OCT), ${ }^{6}$ multiphoton microscopy $^{7,8}$ ) have already been applied for the

*Corresponding author.

This is an Open Access article published by World Scientific Publishing Company. It is distributed under the terms of the Creative Commons Attribution 4.0 (CC-BY) License. Further distribution of this work is permitted, provided the original work is properly cited. 


\section{S. Wu et al.}

diagnosis and delineation of various skin diseases. $^{9-12}$ OCT is a feasible optical biopsy tool with high sensitivity and specificity for noninvasive diagnosis of some skin diseases. ${ }^{6}$ OCT was first put forward by David Huang in 1991 with resolution and penetration depth of $1-15 \mu \mathrm{m}$ and $1-3 \mathrm{~mm}$, respectively. ${ }^{13}$

The parameters of the optical properties which determine the process of optical transmission on tissue have a close relationship with the physiological and pathological status of tissue. The optical characteristics of tissue are critical in photodynamic therapy, medical imaging, monitoring of tissue dynamic. ${ }^{14,15}$ From the view point of tissue optical, the cellular level determines on the optical properties of the physical structure on the biological tissues. ${ }^{16}$ So the image, reflecting the morphological features, could be associated with the optical properties of tissue. In this study, OCT was used to monitor the morphologic changes of the process of UVB induced skin cancer. The epidermis thickness and attenuation coefficient $\left(\mu_{t}\right)$ during the canceration process were extracted by OCT scattering model based on the OCT figures in different time interval of UVB irradiation. ${ }^{16}$ In addition, the local texture features during the canceration process were obtained by gray-level co-occurrence matrix (GLCM) method. ${ }^{17}$ The aim of this study was to analyze the character of the skin cancer process from the perspective of tissue optics and texture feature. It will help us to further understand the characters of the formation process skin cancer.

\section{Materials and Methods}

\subsection{Animal models}

Female ICR mice of five weeks old were obtained from Fuzhou Animal Experiment Center. Animals were kept in a constant temperature and humidity environment with a $12 \mathrm{~h}$ light/dark cycle and fed with standard diet and clean water. A total of 32 mice were randomly assigned to two groups: Group A $(n=8)$ was used for in vivo OCT examination and Group B $(n=24)$ mainly for histological diagnosis at different time points.

To establish the cutaneous SCCs, UVB lamps (280-320 nm, with peak emission at $308 \mathrm{~nm}$ ) were used to irradiate mice in a homemade box. The skin hairs were removed daily prior to UVB irradiation.
The average intensity at the dorsal skin surface was measured and maintained at the same level for each irradiation which was performed three times per week. The irradiation was started with one minute at the beginning and gradually increased to the full length of 30 minutes each time. The mice were irradiated for 22 weeks and the accumulated total dose of UVB was $63.43 \mathrm{~J} / \mathrm{cm}^{2}$. The skin of irradiated mice was examined daily during the course of irradiation. If there were noticeable signs of inflammation and anabrosis, the UVB irradiation would be paused 1 to 3 times until these symptoms relieved. Tumor growth in the irradiated site was examined by an OCT system (Group A) and H.E. staining (Group B), respectively.

\subsection{OCT system and scattering model}

The coherence interferometry produces a twodimensional (2D) image of optical scattering from internal tissue microstructures. Interference fringes are formed when the optical path length of light reflected from the sample matches that reflected from the reference arm within the coherence length of the light source. In this model, the signals were detected by OCT using of ballistic trajectory photons scattered in tissue. The OCT system used in the experiments employed a super-luminescentdiode source, with center wavelength of $842 \mathrm{~nm}$ and bandwidth of $20 \mathrm{~nm} .{ }^{18}$

The light propagating in turbid media obeys the Beer's law as follows:

$$
\varphi(z)=\varphi_{0} \exp \left(-\mu_{t} z\right)
$$

where $\varphi_{0}$ is the influence of incident light, $\mu_{t}$ is the total attenuation coefficient, and $z$ is the medium depth. The optical properties of tissue can be quantified through OCT single scattering model analysis. The availability of the OCT single scattering model is approximately equal to 3-6 mean free paths. ${ }^{18,19}$ The attenuation coefficient $\left(\mu_{t}\right)$ can be fitted using Beer's law from the intensity of detected light vs detectable depth. ${ }^{15}$ The $\mu_{t}$ is extracted from the slope of the OCT signals in logarithmic scale. The method has been applied for characterizing the optical properties and morphological changes of biological tissues. ${ }^{16}$ The procedure developed by David Levitz ${ }^{20}$ was reprogrammed to obtain the attenuation coefficient 
and the epidermis thickness of the skin. The epidermis thickness was obtained from the first peakvalley of the intensity of OCT signal. ${ }^{21}$ While the attenuation coefficient obtained from the OCT intensity using the Beer's law. A region of interest (ROI) of image was selected at first, then, the 2D intensity distributions from each image formed into the single curve to obtain the longitudinal distribution in depth were averaged. Finally, the part of the curve was chosen to fit the one based on Eq. (1) and then the attenuation coefficient $\mu_{t}$ was obtained. ${ }^{20}$

\subsection{Analysis of gray level co-occurrence}

Spatial gray level co-occurrence estimates image properties related to second-order statistics. Gray level co-occurrence matrix has become one of the most well known and widely used texture features. $^{22}$ The greatest strengths of GLCM are the computational ease and power of features. The $G \times G$ gray level co-occurrence matrix $P_{d}$ for a displacement vector $d=(d x, d y)$ is defined as follow. The entry $(i, j)$ of $P$ is the occurrences number of the gray levels pair $i$ and $j$ which are a distance $d$ apart.

$$
\begin{aligned}
P(i, j, d, \theta) & \\
= & \{(x, y),(x+\Delta x, y+\Delta y)] \\
& \left.\mid \frac{f(x, y)=i, f(x+\Delta x, y+\Delta y)=j}{x=0,1, \ldots, N_{x}-1 ; y=0,1, \ldots, N_{y}-1}\right\} .
\end{aligned}
$$

First, an original texture image $\mathrm{D}$ is requantized into an image $G$ with reduced number of gray level, $N_{g}$, a typical value of $N_{g}$ is 16 or 32 . Then, GLCM is computed from $G$ by scanning the intensity of each pixel and its neighbor, defined by displacement $d=$ 20 pixels and angle $\theta=45^{\circ}$.

The skin features were analyzed by GLCM texture to assess the definition of skin structure. Five images from ROI about $1 \mathrm{~mm} \times 0.25 \mathrm{~mm}$, specifically the vertical section of each mouse, were selected for quantitative analysis. The experimental results were analyzed by statistic test using SPSS 15.0 software (SPSS Inc., USA). Statistical significances of the data in different types were evaluated by T-test which is used to determine whether there are any significant differences. The differences were considered statistically significant when the $P$ values obtained from T-test analysis were less than 0.05 .

\section{Results and Discussion}

\subsection{Correlation of OCT image with morphological changes}

The morphological changes from normal skin to SCCs in the UVB-irradiated mouse model were recorded by OCT and histological examination. Figure 1 shows the OCT images (a1-a4) and optical images of biopsy samples (b1-b4) taken at the same position from irradiated mice at four different time points (i.e., 0, 6, 22 and 24 weeks). OCT images showed that the detectable depth of four time points was different. The detectable depth of unirradiated skin was deeper while the optical penetration depth decreased in UVB irradiated skin and SCCs. This suggests that the UVB irradiation of the skin could alter OCT signal intensity. As shown in Fig. 1, there was an obvious cavity between the surface scab and the epithelial cells at six weeks $(a 2)$ and this feature was consistent with the histological images (b2). And then, the skin repeated the process of scabbing and taking off scab. After repeating UVB irradiation, the hyperplastic epidermis became obvious (a3). H.E staining showed that the cavity disappeared (b3), which indicated significant morphological change taking place during the development of cancerous lesions. Continuing irradiation would induce papilloma lesions ( $a 4$ and $b 4$ ). The features of OCT images corresponded well with the histological examination at each time points, which suggests that OCT is a useful tool for identifying cutaneous cancerous changes induced by chronic UVB exposure.

\subsection{Optical parameters}

There are two bright reflective layers identifiable in typical OCT image of the skin; which is useful for determining the thickness of the epidermis. Figure 2 shows the changes in the epidermis thickness over the time. The one extracted from OCT images correlated well with that obtained from H.E staining at different time points although the estimation from OCT was slightly higher than that from H.E staining. The value of epidermis thickness increased with irradiated by UVB. Concretely, the value of 
S. Wu et al.
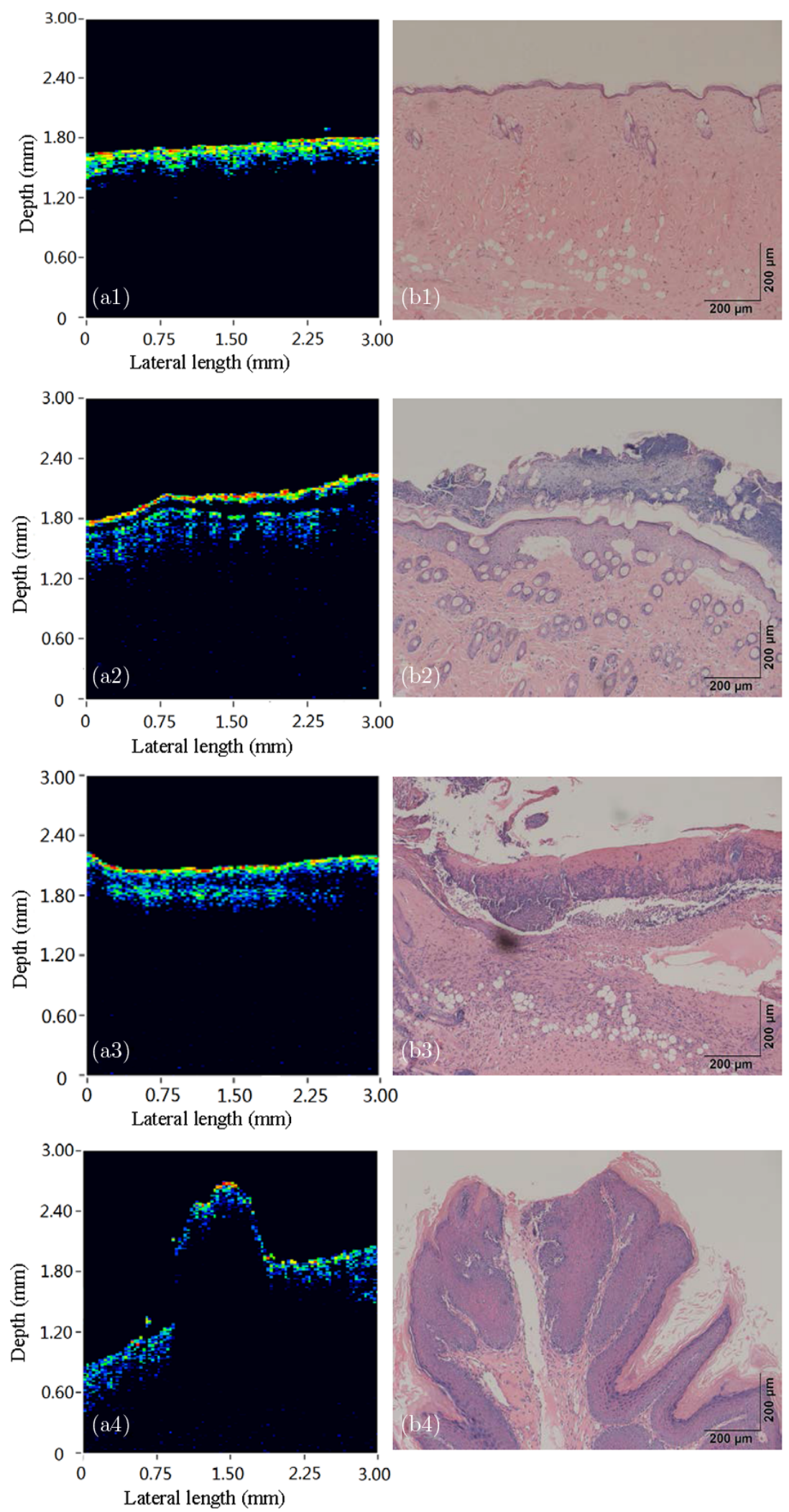

Fig. 1. OCT images and the histological images of different time points. Left panel - OCT image, right panel — histological image. (a1) and (b1): un-irradiated skin; $(a 2)$ and (b2): Six weeks post-irradiation; $(a 3)$ and (b3): 20 weeks post-irradiation; and $(a 4)$ and (b4): 22 weeks post-irradiation. The scale bar is $200 \mu \mathrm{m}$ in H.E staining slice. 


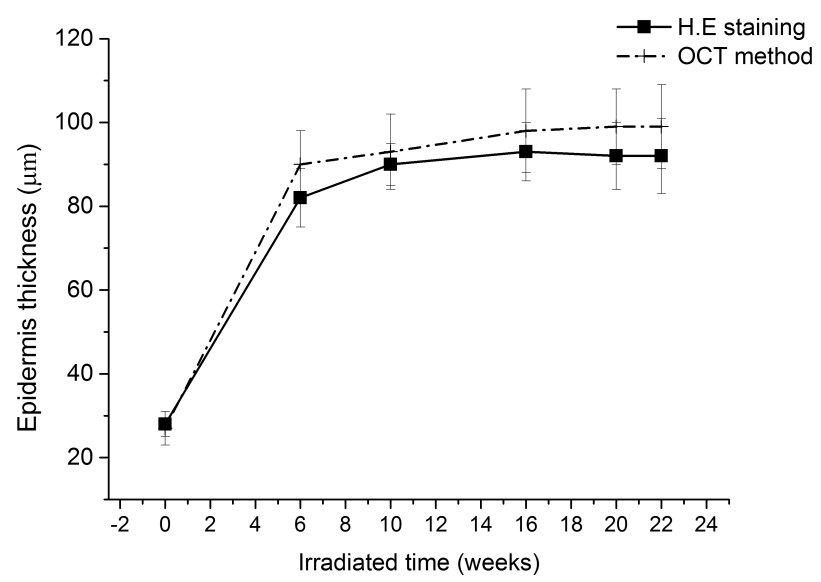

Fig. 2. Measurement of epidermis thickness from H.E. staining method and OCT method.

normal skin was about $28 \mu \mathrm{m}$. After irradiation for six weeks, the thickness of epidermis was about $83 \mu \mathrm{m}$. And then the epidermis thickness maintained a stable value. The outcomes indicated that the OCT method were consistent with the histology method. The changes of epidermis thickness demonstrated that epidermis was injured by UVB irradiated and then scabby.

Moreover, the sizes of the tumor could be extracted from the OCT signal. So OCT is the feasible method to obtain the dimensions of skin tumor or skin injury and the better tool to distinguish the boundary of injured region which would be applied on monitoring the surgical resection.

In order to establish links between the structure changes and the optical parameter changes of the skin during the process of cancerization, OCT single

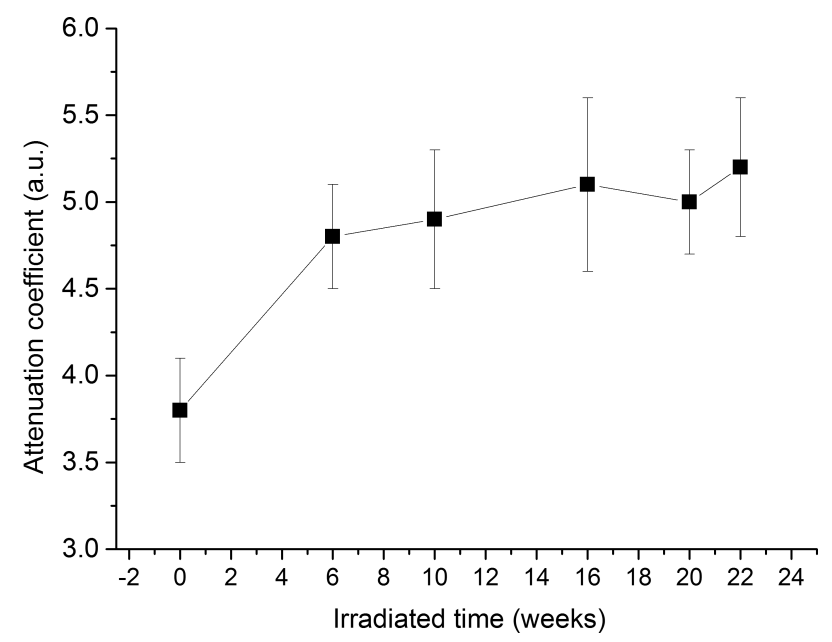

Fig. 3. The attenuation coefficient of skin obtained from OCT scattering model. scattering model was applied to extract and analyze the experimental data. The effective attenuation coefficient $\left(\mu_{t}\right)$ was obtained from OCT intensity figures in the ROI. The attenuation coefficient of six time points was obtained from the whole layer skin. The results are shown in Fig. 3. It is evident that the attenuation coefficient increased with UVB irradiation time. The values were mainly obtained from the epidermis because the epidermis thickness increased as the detectable depth decreased over the time during the process of SCCs development. So the value of attenuation coefficient increased is mainly due to the changes in the epidermis and hemoglobin contents in the epidermis and dermis junction in the presence of inflammation and hyperpigmentation caused by long-term UV irradiation. It is possible that the collagens in the dermis were destroyed to certain degrees and the density of collagen decreased which resulted in low scattering in the irradiated skin tissue.

\subsection{Texture feature}

The speckle pattern of OCT image may be regarded as the result of the coherent superposition of the interference fringes falling into the observation plane. The $2 \mathrm{D}$ speckle could be considered as the tissue texture. The texture features of OCT images could reflect the skin characteristics from longitudinal angle. ${ }^{23}$

Based on GLCM method, three texture features, i.e., energy, entropy and correlation were extracted on the OCT image using ROI of $1 \mathrm{~mm} \times 0.25 \mathrm{~mm}$ as shown in Fig. 4(a). Specifically, the energy reflects the roughness of the texture. ${ }^{22}$ The results of normalized energy of longitudinal skin on different time points are shown in Fig. 4(b). The value of the energy increased over the time. In particular, in the earlier stage, the value increased sharply. Later, the value increased slowly. This result implies that the skin has been damaged after a few weeks of irradiation, which results in structural change. Under the cumulative dose, cancerous changes could be developed. In this process, the skin texture became rougher with time. It indicated that the texture of OCT image was distinct in normal skin and the pixel matrix became different after UVB irradiation. Over the time, the intensity of the OCT signal between epidermis and air interface changed greatly. The distribution of light in tissue was not uniform, so its penetration depth also varied. This 


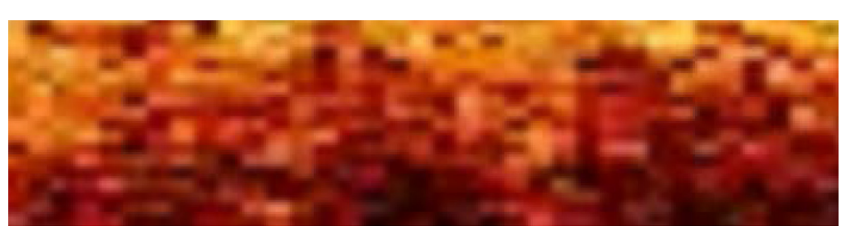

(a)

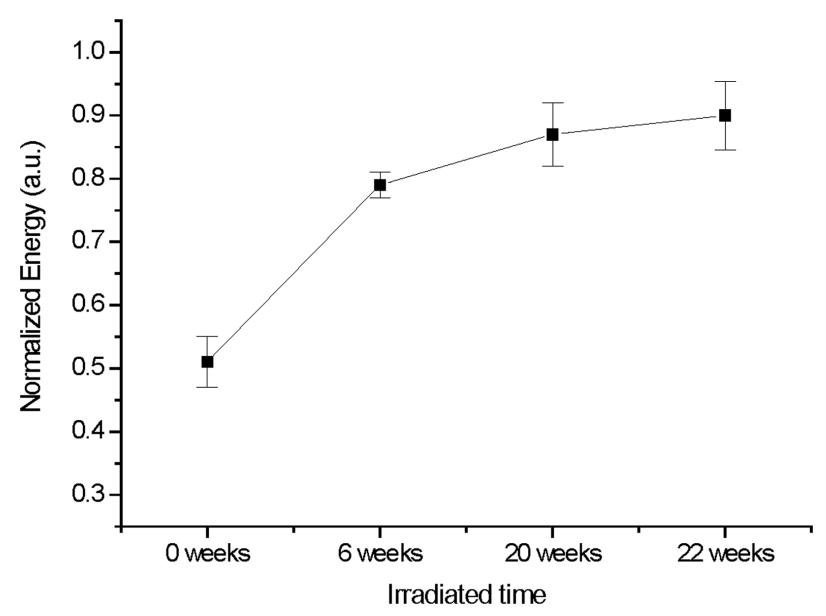

(b)

Fig. 4. (a) The region of OCT image for texture analysis, the size is about $1 \mathrm{~mm} \times 0.25 \mathrm{~mm}$; (b) the energy of skin in OCT image at different time points.

suggested that in the process of UVB irradiation, the corresponding energy increased gradually as the skin barrier was destroyed and the skin texture became coarse.

Entropy is the complexity feature of an image matrix and it mainly reflects the texture grayscale randomness distribution of the image. Entropy is a

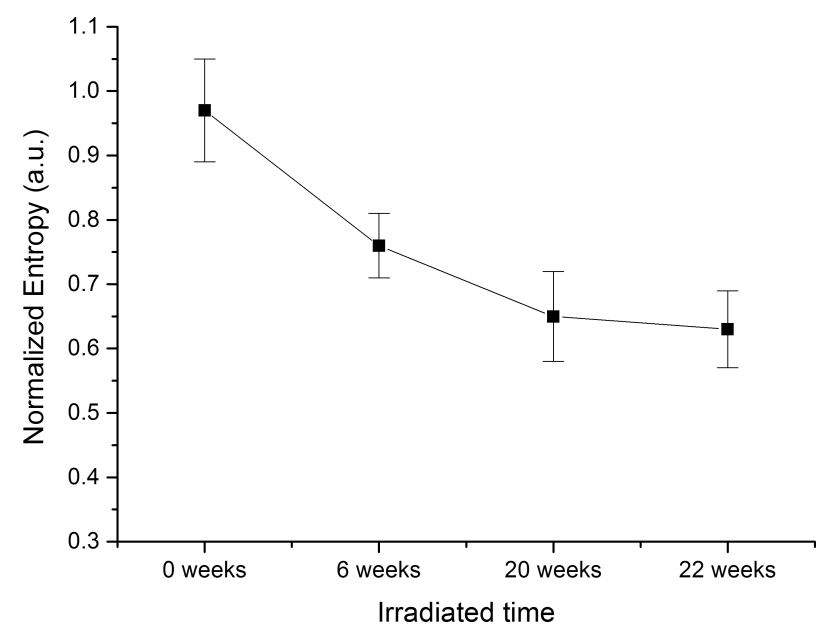

Fig. 5. The entropy of skin at different time points.

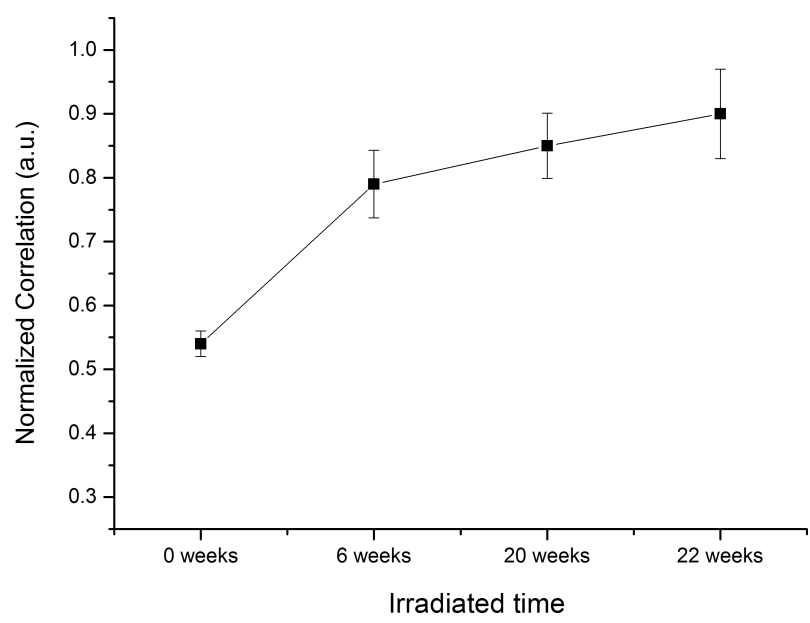

Fig. 6. The correlation at different time points.

measure of the amount of information in an image. $^{22}$ The entropy values in skin at different UVB doses are shown in Fig. 5. The entropy value in un-irradiated skin was greater than those irradiated. This result indicated that the normal skin texture had many fine textures and linear fibrils, whereas the irradiated skin had less fine textures. Thus, texture was complex in normal skin and simple in damage skin.

The correlation of the images reflects the similarity on a direction of the image texture area and is the linear correlation measure of the local gray level in the image. The correlation is a measure of the dependence between two different pixel values. ${ }^{22}$ The skin correlativity in the process of UVB induced tumor is shown in Fig. 6. The skin correlation value in irradiated skin was larger than that in normal skin, indicating that injure skin texture had faint fibrils and a uniform pixel matrix. The value of skin correlation could be used to quantitatively estimate the similarity among various time points. The extent of the similarity depends on the correlation value. It indicates that the more similar, the more UVB-induced injure. Moreover, it changed more dramatically at the early stage of irradiation.

\section{Conclusions}

In this study, the process of SCC induced by UVB was monitored by OCT technology in vivo and compared with the histology analysis - H.E stained slice. The morphologic structure, optical characteristics, and texture features were obtained 
from OCT image to study the skin changes in the SCC formation. The detectable depth decreased with the irradiated time. The epidermis thickness increased with irradiated time caused by the injured skin and with scab. And then, the $\mu_{t}$ had a rising trend in the skin, the main reason was injured epidermis and increased hemoglobin in the junction epidermis. Three main parameters, energy, entropy, and correlation were investigated to determine the roughness, complexity, and similarity of the skin during the SCC formation process, respectively. The results indicated that the complexity in terms of un-irradiation skin texture distribution. By contrast, with the irradiation time, skin was blurred and had a uniform pixel matrix. All these results obviously suggest that OCT is a feasible technology to monitor the process of SCC and diagnose the skin diseases in vivo. And the result described the SCC process from the perspective of tissue optics and texture feature. It will help us to understand the characters of the formation process skin cancer.

\section{Acknowledgment}

This work was supported by the National Natural Science Foundation of China (61178089), the Natural Science Foundation of Fujian Province (2014J01226) and Foundation of Fujian Educational Committee (JA14093).

\section{References}

1. T. Diepgen, V. Mahler, "The epidemiology of skin cancer," Brit. J. Dermatol. 146, 1-6 (2002).

2. D. Narayanan, R. Saladi, J. Fox, "Review: Ultraviolet radiation and skin cancer," Int. J. Dermatol. 49, 978-986 (2010).

3. R. Siegel, J. Ma, Z. Zou, A. Jemal, "Cancer statistics, 2014, CA: A cancer journal for clinicians," 64, 9-29 (2014).

4. U. Leiter, C. Garbe, "Epidemiology of melanoma and nonmelanoma skin cancer — the role of sunlight, in Sunlight, vitamin D and skin cancer," Adv. Clin. Exp. Med. 624, 89-103 (2008).

5. J. Li, Z. Liu, C. Tan, X. Guo, L. Wang, A. Sancar, D. Zhong, "Dynamics and mechanism of repair of ultraviolet-induced (6-4) photoproduct by photolyase," Nature 466, 887-890 (2010).

6. E. Sattler, R. Kästle, J. Welzel, "Optical coherence tomography in dermatology," J. Biomed. Opt. 18, 061224-061224 (2013).
7. E. Yew, C. Rowlands, P. So, "Application of multiphoton microscopy in dermatological studies: A mini-review," J. Innov. Opt. Health Sci. 7, 1330010 (2014).

8. C. Longo, E. Moscarella, G. Argenziano, A. Lallas, M. Raucci, G. Pellacani, A. Scope, "Reflectance confocal microscopy in the diagnosis of solitary pink skin tumors: Review of Diagnostic Clues," Brit. J. Dermatol. 173, 31-41 (2015).

9. V. Korde, G. Bonnema, W. Xu, C. Krishnamurthy, J. Ranger-Moore, K. Saboda, L. Slayton, S. Salasche, J. Warneke, D. Alberts, "Using optical coherence tomography to evaluate skin sun damage and precancer," Laser Surg. Med. 39, 687-695 (2007).

10. B. Vakoc, D. Fukumura, R. Jain, B. Bouma, "Cancer imaging by optical coherence tomography: Preclinical progress and clinical potential," Nature Rev. Cancer 12, 363-368 (2012).

11. Z. Liu, Z. Guo, Z. Zhuang, J. Zhai, H. Xiong, C. Zeng, "Quantitative optical coherence tomography of skin lesions induced by different ultraviolet B sources," Phys. Med. Biol. 55, 6175-6185 (2010).

12. T. Gambichler, V. Jaedicke, S. Terras, "Optical coherence tomography in dermatology: Technical and clinical aspects," Arch. Dermatol. Res. 303, 457-473 (2011).

13. D. Huang, E. A. Swanson, C. P. Lin, J. S. Schuman, W. G. Stinson, W. Chang, M. R. Hee, T. Flotte, K. Gregory, C. A. Puliafito, "Optical coherence tomography," Science 254, 1178-1181 (1991).

14. S. Jacques, "Optical properties of biological tissues: A review," Phys. Med. Biol. 58, R37-R61 (2013).

15. W. Cheong, S. A. Prahl, A. J. Welch, "A review of the optical properties of biological tissues," IEEE J. Quantum Elec. 26, 2166-2185 (1990).

16. E. Cauberg, D. de Bruin, D. Faber, T. de Reijke, M. Visser, J. de La Rosette, T. van Leeuwen, "Quantitative measurement of attenuation coefficients of bladder biopsies using optical coherence tomography for grading urothelial carcinoma of the bladder," J. Biomed. Optics. 15, 066013-066016 (2010).

17. C. Honeycutt, R. Plotnick, "Image analysis techniques and gray-level co-occurrence matrices (GLCM) for calculating bioturbation indices and characterizing biogenic sedimentary structures," Comput. Geosci. UK 34, 1461-1472 (2008).

18. J. Schmitt, A. Knüttel, M. Yadlowsky, M. Eckhaus, "Optical-coherence tomography of a dense tissue: Statistics of attenuation and backscattering," Phys. Med. Biol. 39, 1705-1720 (1994).

19. J. Schmitt, A. Knüttel, R. Bonner, "Measurement of optical properties of biological tissues by lowcoherence reflectometry," Appl. Opt. 32, 6032-6042 (1993). 
S. Wu et al.

20. P. Tomlins, R. Wang, "Theory, developments and applications of optical coherence tomography," J. Phys. D, Appl. Phys. 38, 2519-2535 (2005).

21. S. Wu, H. Li, X. Zhang, Z. Li, "Optical features for chronological aging and photoaging skin by optical coherence tomography," Laser Med. Sci. 28, 445-450 (2013).
22. R. Haralick, K. Shanmugam, I. Dinstein, "Textural features for image classification," IEEE T. Syst. Man CY-S. 6, 610-621 (1973).

23. K. Gossage, T. Tkaczyk, J. Rodriguez, J. Barton, "Texture analysis of optical coherence tomography images: Feasibility for tissue classification," J. Biomed. Opt. 8, 570-575 (2003). 